\title{
THE
}

7-18-1983

\section{Dynamic Correlation Functions for Quantum Spin Chains}

Gerhard Müller

University of Rhode Island, gmuller@uri.edu

Robert E. Shrock

Follow this and additional works at: https://digitalcommons.uri.edu/phys_facpubs

Terms of Use

All rights reserved under copyright.

\section{Citation/Publisher Attribution}

Gerhard Müller and Robert E. Shrock. Dynamic correlation functions for quantum spin chains. Phys. Rev. Lett. 51 (1983), 219-222.

Available at: http://dx.doi.org/10.1103/PhysRevLett.51.219

This Article is brought to you for free and open access by the Physics at DigitalCommons@URI. It has been accepted for inclusion in Physics Faculty Publications by an authorized administrator of DigitalCommons@URI. For more information, please contact digitalcommons-group@uri.edu. 


\title{
Dynamic Correlation Functions for Quantum Spin Chains
}

\author{
Gerhard Müller and Robert E. Shrock \\ Institute for Theoretical Physics, State University of New York at Stony Brook, Stony Brook, New York 11794
}

(Received 24 May 1983)

\begin{abstract}
New exact results are presented on the long-time asymptotic expansions of the $T=0$ autocorrelation functions, and on the leading singularities of their frequency-dependent Fourier transforms, for the one-dimensional $S=\frac{1}{2}$ isotropic $X Y$ model and the $S=\frac{1}{2}$ transverse Ising model at the critical field. High-precision numerical calculations of the latter functions are also given, and experiments are proposed to observe the functional behaviors that are found.
\end{abstract}

PACS numbers: $75.10 . \mathrm{Jm}, 75.40 . \mathrm{Fa}$

Exactly solvable models of one-dimensional (1D) spin systems have contributed greatly to our present understanding of statistical mechanics and condensed-matter physics. Although the thermodynamic properties of those models have been calculated, very little is known about the dynamic correlation functions. However, the knowledge of these dynamic correlation functions is crucial for understanding the excitation spectrum of the underlying model and its observability in dynamical experiments.

In this Letter we study the dynamics at temperature $T=0$ of the $1 \mathrm{D}, S=\frac{1}{2}$ transverse Ising (TI) model at the critical external magnetic field and the $1 \mathrm{D}, S=\frac{1}{2}$ isotropic $X Y$ model in zero field, defined by the respective Hamiltonians

$$
\begin{aligned}
& H_{\mathrm{TI}}=-\sum_{l=1}^{N}\left(2 S_{l}{ }^{x} S_{l+1}{ }^{x}+h_{c} S_{l}{ }^{z}\right), \quad h_{c}=1, \\
& H_{X Y}=-\sum_{l=1}^{N}\left(S_{l}{ }^{x} S_{l+1}{ }^{x}+S_{l}{ }^{y} S_{l+1}{ }^{y}\right),
\end{aligned}
$$

in the limit $N \rightarrow \infty$, with periodic boundary conditions imposed. We report here the first calculation of the Fourier-transformed spin autocorrelation functions (ACF's)

$$
\Phi_{0}^{\mu \mu}(\omega)=\int_{-\infty}^{\infty} d t e^{i \omega t}\left\langle S_{0}{ }^{\mu}(t) S_{0}{ }^{\mu}\right\rangle
$$

for $\mu=x, y$, in these models, based on an exact approach. Our results are of direct experimental interest, since there are several compounds which exhibit quasi-1D interactions described by (1) or (2). ${ }^{1} \Phi_{0}{ }^{\mu \mu}(\omega)$ is important since it is the integral over $q$ of the dynamic structure factor $S_{\mu \mu}(q, \omega)$ measured by inelastic neutron scattering, and, for small $\omega$, is related to the spinlattice relaxation rate measured by nuclear magnetic resonance experiments.

We have analytically determined the singularities in $\Phi_{0}{ }^{\mu \mu}(\omega)$ by means of a newly extended longtime asymptotic expansion (LTAE) at $T=0$ of the ACF's $X_{0}(t)$ and $Y_{0}(t)$ [we use the notation
$\Xi_{n}(t) \equiv 4\left\langle S_{0}{ }^{\xi}(t) S_{n}{ }^{\xi}\right\rangle, \xi=x, y, z$ for the two-spin correlation functions]. $Z_{n}(t)$ has been known analytically for many years for both models at arbitrary $T_{0}{ }^{2}$ In contrast, there has never been any complete calculation of $X_{n}(t)$ or $Y_{n}(t)$ for general $T$ and, in particular, for the case $T=0$ of interest here. ${ }^{3}$ This can be understood as a result of the fact that, after the Jordan-Wigner transformation from spin operators to fermion operators, $Z_{n}(t)$ involves only a product of four fermion operators, whereas $X_{n}(t)$ and $Y_{n}(t)$ involve an infinite number of these operators. The latter ACF's are thus much more complicated objects and represent (in the fermion language) not just the excitation of particle-hole pairs, but rather the excitation of arbitrarily many such pairs.

Recently, progress has been made in determining $X_{n}(t)$ and $Y_{n}(t)$ at $T=0$ for both models. It has been shown ${ }^{4}$ that $\left[X_{n}(t)\right]_{\mathrm{TI}}$ can be expressed in terms of a function $\sigma_{n}(z)$ as

$$
\begin{aligned}
& {\left[X_{n}(t)_{\mathrm{TI}}\right.} \\
& =\left[X_{n}(0)\right]_{\mathrm{TI}} \exp \left[-t^{2} / 2+\int_{0}^{2 t} d t^{\prime} t^{\prime-1} \sigma_{n}\left(i t^{\prime}\right)\right],
\end{aligned}
$$

where $\left[X_{0}(0)\right]_{\mathrm{TI}}=1$ and, for $|n| \geqslant 1$,

$$
\left[X_{n}(0)\right]_{\mathrm{TI}}=(2 / \pi)^{|n|} \prod_{l=1}^{|n|}\left[1-1 /(2 l)^{2}\right]^{l-|n|}
$$

and $\sigma_{n}(z)$ satisfies

$$
\left(z \sigma_{n}{ }^{\prime \prime}\right)^{2}+4\left(z \sigma_{n}{ }^{\prime}-\sigma_{n}-n^{2}\right)\left[z \sigma_{n}{ }^{\prime}-\sigma_{n}+\left(\sigma_{n}{ }^{\prime}\right)^{2}\right]=0
$$

with the initial condition that for $z \rightarrow 0$

$$
\sigma_{n}(z)=\sum_{k=1}^{\infty} a_{k, n} z^{2 k}+z^{2 n+1} \sum_{k=0}^{\infty} b_{k, n} z^{2 k}
$$

where all $a_{k, n}$ and $b_{k, n}$ can be calculated recursive ly in terms of $b_{0, n}$, and for the ACF $(n=0), b_{0,0}$ $=1 / \pi$. Given $\left[X_{0}(t)\right]_{\mathrm{TI}}$, the other ACF's of interest can be calculated from it by means of the relations $^{5}\left[Y_{0}(t)\right]_{\mathrm{TI}}=-\left[\ddot{X}_{0}(t)\right]_{\mathrm{TI}}$ and $^{6}\left[X_{0}(t)\right]_{X Y}$ $=\left[Y_{0}(t)\right]_{X Y}=\left\{\left[X_{0}(t / 2)\right]_{\mathrm{TI}}\right\}^{2}$. 
For Ref. 4, Eq. (5) was solved numerically for $\boldsymbol{n}=0$, and from this numerical solution an anatic Ansatz for the LTAE of $\sigma_{0}(i t)$ was inferred, and then verified analytically. This LTAE was calculated for general $b_{0,0}$ to $O\left(t^{-1}\right)$ and, with some further terms for the physical value $b_{0,0}=1 / \pi$, was used to construct the resultant LTAE of
$\left[X_{0}(t)\right]_{\mathrm{TI}}$ to $O\left(t^{-11 / 4}\right)$. For our present purposes we have extended the LTAE of $\sigma_{0}(i t)$ completely to $O\left(t^{-3}\right)$ and have calculated the terms of $O\left(t^{-7 / 2}\right)$ that are necessary to obtain the leading $e^{-6 i t}$ term in $\left[X_{0}(t)\right]_{\mathrm{TI}}$, which enters first at $O\left(t^{-19 / 4}\right)$. We find that the LTAE of $\left[X_{0}(t)\right]_{\text {TI }}$ has the following structure, as far as we have determined it:

$$
\left[X_{0}(t)\right]_{\mathrm{TI}} \sim \bar{A}(i t)^{-1 / 4} \sum_{m=0}^{\infty} T_{x, m}{ }^{(\mathrm{TI})}, \quad T_{x, m}{ }^{(\mathrm{TI})}=(2 \pi)^{-m / 2} e^{-2 i m t}(-2 i t)^{-\alpha_{m}} 2^{-\beta_{m}} \sum_{n=0}^{\infty} c_{n}{ }^{(m)}(-2 i t)^{-n} .
$$

Here $\bar{A}=2^{1 / 12} \exp \left[3 \zeta^{\prime}(-1)\right]$, the coefficients $c_{n}{ }^{(m)}$ are positive rational numbers with $c_{0}{ }^{(m)}=1$ for all $m$ and $c_{2 n+1}{ }^{(0)}=0$ for all $n$, and the exponents $\alpha_{m}$ are positive integers or half-integers. The values of $c_{n}{ }^{(m)}, \alpha_{m}$, and $\beta_{m}$ which have so far been calculated are listed in Table I. We have written the LTAE of $\left[X_{0}(t)\right]_{\text {TI }}$ in a form which renders its well-known symmetry property $X_{0} *(-t)=X_{0}(t)$ manifest.

Further general features of this LTAE are the following:

(i) The dominant term at large $t$ is $\bar{A}(i t)^{-1 / 4}$. This reflects the Lorentz invariance in a scaling limit, given the result that ${ }^{7}\left[X_{n}(0)\right]_{\mathrm{TI}} \sim \bar{A} n^{-1 / 4}$, for large $n$.

(ii) $\left[X_{0}(t)\right]_{\mathrm{TI}}$ consists of an infinite sum of terms $T_{x, m}{ }^{(\mathrm{TI})}, m=0,1 . \ldots$, each with a specific oscillatory $t$ dependence given by the phase factor $e^{-2 i m t}$. The fact that all the terms in (7) have frequencies of only one sign is a consequence of the detailed balance condition, which implies that at $T=0$, $\Phi_{0}{ }^{\mu \mu}(\omega)=0$ for $\omega<0$.

(iii) Each term $T_{x, m}{ }^{\text {(TI) }}$ itself is an infinite series of terms with descending powers of $t$ and will thus be denoted as a "tower." Each successive tower enters first at a progressively higher level in the expansion; i.e., the higher the oscillation

TABLE I. Coefficients $c_{n}^{(m)}, \alpha_{m}$, and $\beta_{m}$ of the LTAE of $\left[X_{0}(t)\right]_{\mathrm{TI}}$; coefficents $d_{n}^{(m)}, \gamma_{m}$, and $\delta_{m}$ of the LTAE of $\left[X_{0}(t)\right]_{\mathbf{X Y}}$.

\begin{tabular}{|c|c|c|}
\hline $\begin{array}{l}c_{2}{ }^{(0)}=2^{-3} \\
c_{4}{ }^{(0)}=(81 / 2)^{7} \\
c_{1}{ }^{(1)}=(9 / 2)^{3} \\
c_{2}{ }^{(1)}=(297 / 2)^{7} \\
c_{3}{ }^{(1)}=(7587 / 2)^{10}\end{array}$ & $\begin{aligned} c_{1}^{(2)} & =(15 / 2)^{2} \\
c_{2}^{(2)} & =(489 / 2)^{5} \\
\alpha_{0} & =0 \\
\alpha_{1} & =1 / 2 \\
\alpha_{2} & =2\end{aligned}$ & $\begin{array}{l}\alpha_{3}=9 / 2 \\
\beta_{0}=0 \\
\beta_{1}=0 \\
\beta_{2}=2 \\
\beta_{3}=5\end{array}$ \\
\hline $\begin{array}{l}d_{2}^{(0)}=2^{-2} \\
d_{4}^{(0)}=(41 / 2)^{5} \\
d_{1}^{(1)}=(9 / 2)^{3} \\
d_{2}^{(1)}=(313 / 2)^{7} \\
d_{3}^{(1)}=(7731 / 2)^{10} \\
d_{1}^{(2)}=(11 / 2)^{2}\end{array}$ & $\begin{aligned} d_{2}^{(2)} & =(249 / 2)^{5} \\
d_{3}^{(2)} & =(3551 / 2)^{7} \\
d_{1}^{(3)} & =(39 / 2)^{3} \\
d_{2}^{(3)} & =(2809 / 2)^{7} \\
\gamma_{0} & =0 \\
\gamma_{1} & =1 / 2\end{aligned}$ & $\begin{array}{l}\gamma_{2}=1 \\
\gamma_{3}=5 / 2 \\
\delta_{0}=0 \\
\delta_{1}=-1 \\
\delta_{2}=0 \\
\delta_{3}=1\end{array}$ \\
\hline
\end{tabular}

frequency, the more highly suppressed the tower is in $t$.

The LTAE of $\left[Y_{0}(t)\right]_{\text {TI }}$ as calculated from (7) is again an infinite sum of towers, but differs from $\left[X_{0}(t)\right]_{\mathrm{TI}}$ in that, for example, the leading term comes not from $T_{y, 0}{ }^{(T I)}$ but from $T_{y, 1}{ }^{\text {(TI) }}$ and is $\bar{A} 2^{3 / 2} e^{-2 i t}(i t)^{-1 / 4}(-i t)^{-1 / 2}$. Hence, a continuum approximation would fail completely for $\left[\boldsymbol{Y}_{0}(t)\right]_{\mathrm{TI}}$, since it would predict that the leading term at large $t$ is $\propto t^{-9 / 4}$, corresponding to the $n^{-9 / 4}$ behavior ${ }^{7}$ of $\left[Y_{n}(0)\right]_{\mathrm{TI}}$ for large $n$; the latter is correct only for the discrete system. The fact that $\left[Y_{0}(t)\right]_{\mathrm{TI}}$ falls off more rapidly at large $t$ than $\left[X_{0}(t)\right]_{\mathrm{TI}}$ reflects the fact that the $x$ and $y$ axes are the "easy" and the "hard" spin-fluctuation directions, respectively.

In order to determine $\left[\Phi_{0}^{x x}(\omega)\right]_{\mathrm{TI}}$, we have used the numerical calculation of $\left[X_{0}(t)\right]_{\mathrm{TI}}$ which was carried out for Ref. 4. At $t=40$, this solution matches the known LTAE to within a relative error of $10^{-6}$. We generate $\left[\Phi_{0}^{x x}(\omega)\right]_{\mathrm{TI}}$ by a fastFourier-transform program using the precise numerical results for $\left[X_{0}(t)\right]_{\mathrm{TI}}$ for $t<40$ and the LTAE for $t>40$. The resulting $\left[\Phi_{0}^{x x}(\omega)\right]_{\mathrm{TI}}$ is

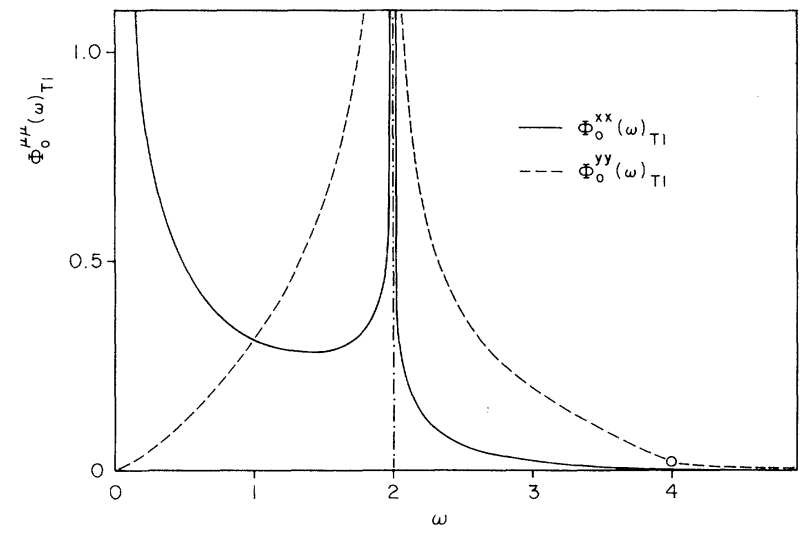

FIG. 1. The frequency-dependent autocorrelation functions $\left[\Phi_{0}{ }^{x x}(\omega)\right]_{\mathrm{TI}}$ and $\left[\Phi_{0}{ }^{y y}(\omega)\right]_{\mathrm{TI}}$. The dominant singularities at $\omega=0,2,4$ are given in Eq. (8). 
plotted in Fig. 1. The accuracy of the curve is better than 1 part in $10^{3}$ over the range of $\omega$ shown. We also give $\left[\Phi_{0}{ }^{y y}(\omega)\right]_{\mathrm{TI}}=\omega^{2}\left[\Phi_{0}{ }^{x x}(\omega)\right]_{\mathrm{TI}}$ in Fig. 1.

These frequency-dependent ACF's evidently have quite striking behavior including "visible" singularities at $\omega=0,2,4$. From the LTAE of $\left[X_{0}(t)\right]_{\mathrm{TI}}$ we find that the dominant singularities of $\left[\Phi_{0}{ }^{x x}(\omega)\right]_{\mathrm{TI}}$ at $\omega=0,2,4$ are given, respectively, by

$$
\left[\Phi_{0}^{x x}(\omega)\right]_{\mathrm{TI}} \sim\left\{\begin{array}{l}
2^{-3 / 2} \bar{A} \Gamma\left(\frac{3}{4}\right) \omega^{-3 / 4} \theta(\omega), \\
2^{-2} \pi^{-1 / 2} \bar{A} \Gamma\left(\frac{1}{4}\right)|\omega-2|^{-1 / 4}\left\{\theta(2-\omega)+2^{-1 / 2} \theta(\omega-2)\right\}, \\
2^{-13 / 2} \pi^{-1} \bar{A} \Gamma\left(-\frac{5}{4}\right)(\omega-4)^{5 / 4} \theta(\omega-4) .
\end{array}\right.
$$

The leading singularities of $\left[\Phi_{0}^{y y}(\omega)\right]_{\mathrm{TI}}$ are of the same form as those of $\left[\Phi_{0}^{x x}(\omega)\right]_{\mathrm{TI}}$ except the one at $\omega=0$, which is $\sim \omega^{5 / 4} \theta(\omega)$. The difference in the singularity at $\omega=0$ can be attributed to the fact that $x$ is the "easy" and $y$ the "hard" spin-fluctuation direction. The LTAE's indicate that $\left[\Phi_{0}{ }^{x x}(\omega)\right]_{\mathrm{TI}}$ and $\left[\Phi_{0}{ }^{y y}(\omega)\right]_{\mathrm{TI}}$ should have finite points of nonanalyticity at $\omega=2 m, m \geqslant 2$ whose dominant terms are of the form $(\omega-2 m)^{\nu_{m}} \theta(\omega-2 m)$ or $|\omega-2 m|^{\nu} m\left\{\theta(2 m-\omega) \pm 2^{-1 / 2} \theta(\omega-2 m)\right\}$, where $\nu_{m}$ is a monotonically increasing function of $m$. We find that both $\left[\Phi_{0}{ }^{x x}(\omega)\right]_{\mathrm{TI}}$ and $\left[\Phi_{0}{ }^{y y}(\omega)\right]_{\mathrm{TI}}$ decrease quite rapidly to zero at $\omega>2$. In fact, sum rules require that as $\omega \rightarrow \infty$, these functions tend to zero faster than any power. ${ }^{8}$

From (7) we calculate the LTAE of $\left[X_{0}(t)\right]_{X Y}$ :

$$
\left[X_{0}(t)\right]_{X Y} \sim(\bar{A})^{2} 2^{1 / 2}(i t)^{-1 / 2} \sum_{m=0}^{\infty} T_{x, m}{ }^{(X Y)}, \quad T_{x, m}{ }^{(X Y)}=(2 \pi)^{-m / 2} e^{-i m t}(-i t)^{-\gamma_{m}} 2^{-\delta_{m}} \sum_{n=0}^{\infty} d_{n}^{(m)}(-i t)^{-n} .
$$

The coefficients $d_{n}{ }^{(m)}$ are again positive rational numbers with $d_{0}{ }^{(m)}=1$ for all $m$ and $d_{2 n+1}{ }^{(0)}=0$ for all $n$. The values of $d_{n}{ }^{(m)}, \gamma_{m}$, and $\delta_{m}$ which have been calculated are listed in Table I. The leading term in (9), which is $\propto t^{-1 / 2}$, can again be understood as reflecting Lorentz invariance in a scaling limit, given the result ${ }^{9}$ that $\left[X_{n}(0)\right]_{X Y}$ $\sim n^{-1 / 2}$ for large $n$. In contrast to the TI case, all nonnegative integral frequencies, not only the even ones, occur. As with $\left[X_{0}(t)\right]_{T 1}$, the higherfrequency towers enter at more highly suppressed levels in $t$.

By the same method as for the TI model, and with equally high precision, we have numerically calculated $\left[\Phi_{0}{ }^{x x}(\omega)\right]_{X Y}$. Our result is plotted in Fig. 2. From the LTAE (9) we find that the dominant singularities in $\left[\Phi_{0}{ }^{x x}(\omega)\right]_{X Y}=\left[\Phi_{0}{ }^{y y}(\omega)\right]_{X Y}$ at $\omega=0,1,2$ are, respectively,

$$
\left[\Phi_{0}{ }^{x x}(\omega)\right]_{X Y} \sim\left\{\begin{array}{l}
(\bar{A})^{2} 2^{-1 / 2} \pi^{1 / 2} \omega^{-1 / 2} \theta(\omega), \\
(\bar{A})^{2} 2^{-1} \pi^{-1 / 2} \ln \left[\left(\frac{1+\omega}{1-\omega}\right)^{2}\right], \quad(10) \\
-(\bar{A})^{2} 2^{-1 / 2} \pi^{-1 / 2}(\omega-2)^{1 / 2} \theta(\omega-2) .
\end{array}\right.
$$

These singularities are clearly visible in Fig. 2. The LTAE indicates that $\left[\Phi_{0}{ }^{x x}(\omega)\right]_{X Y}$ has further finite points of nonanalyticity at all integral frequencies $\omega>2$, with monotonically increasing positive rational powers, similar to the TI case.

Since the $x-y$ plane is the "easy" plane in the $\boldsymbol{X Y}$ model and the $x$ axis is the "easy" axis of the TI model for spin fluctuations, it is appropriate to compare $\left[\Phi_{0}^{x x}(\omega)\right]_{X Y}$ and $\left[\Phi_{0}^{x x}(\omega)\right]_{\mathrm{TI}}$. We inter pret the greater severity of the $\omega=0$ singularity in $\left[\Phi_{0}{ }^{x x}(\omega)\right]_{\mathrm{TI}}$ relative to that in $\left[\Phi_{0}{ }^{x x}(\omega)\right]_{X Y}$ as reflecting the greater extent of spin fluctuations in the $X Y$ model. This is an accord with the wellknown fact that the static correlations at large distances are stronger in the TI model $\left(\sim n^{-1 / 4}\right)$ relative to the $X Y$ model $\left(\sim n^{-1 / 2}\right) .^{9}$

A further noteworthy finding is that $\left[\Phi_{0}{ }^{\mu \mu}(\omega)\right]_{\mathrm{TI}}$ and $\left[\Phi_{0}{ }^{\mu \mu}(\omega)\right]_{X Y}, \mu=x, y$, are convex functions of $\omega$ on the intervals between each of their respective singularities.

Finally, we emphasize that the divergences and

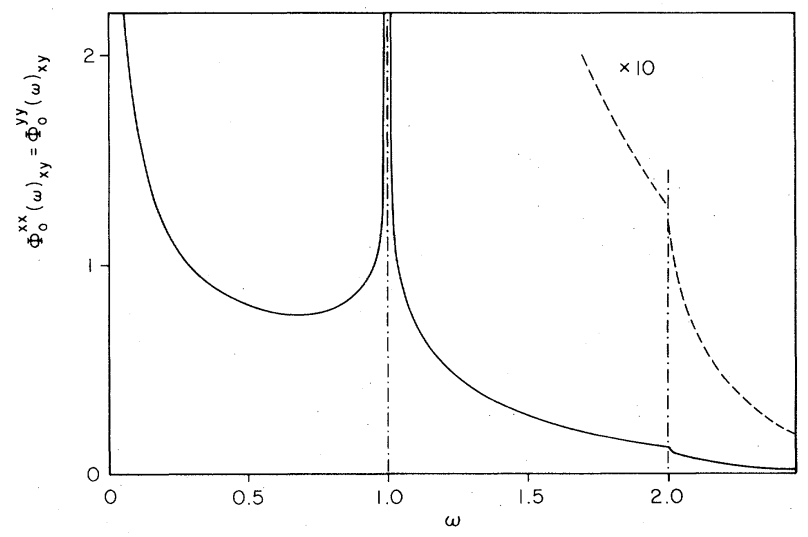

FIG. 2. The frequency-dependent autocorrelation function $\left[\Phi_{0}{ }^{x x}(\omega)\right]_{X Y}=\left[\Phi_{0}{ }^{y y}(\omega)\right]_{X Y}$. The dominant singularities at $\omega=0,1,2$ are given in Eq. (10). 
finite nonanalyticities in the ACF's at nonzero frequencies are intrinsic features of the discrete quantum chain and therefore would not be found by any continuum analysis. ${ }^{10}$

In summary, we have extended the long-time asymptotic expansions of the zero-temperature autocorrelation functions for the $1 \mathrm{D}, S=\frac{1}{2}$ transverse Ising model at the critical field and the $1 \mathrm{D}$, $S=\frac{1}{2}$ isotropic $X \boldsymbol{Y}$ model at zero field. We have combined these new analytic results with the numerical solution to the ordinary differential equation governing the time dependence of the ACF's to calculate by Fourier transform the frequencydependent ACF's. Finally, we have determined the exact form of the singularities in the latter functions.

Our new results provide strong motivation for experimentalists to measure the low-temperature dynamical properties of quasi-1D compounds to observe the frequency behaviors shown in Figs. 1 and 2 (realistically smeared). Such compounds include, for example, the $S=\frac{1}{2} X Y$-like substances $\mathrm{Cs}_{2} \mathrm{CoCl}_{4}$ and $\mathrm{PrCl}_{3}$ and the $S=\frac{1}{2}$ Isinglike substance $\mathrm{CsCoCl}_{3} \cdot 2 \mathrm{H}_{2} \mathrm{O}$.

We thank B. McCoy and J. Perk for useful discussions. This research was supported in part by National Science Foundation Grant No. PHY 81-09110.

${ }^{1}$ For early and recent reviews, see, respectively, M. Steiner, J. Villain, and C. Windsor, Adv. Phys. 25, 87 (1976); and H. Beck, M. Puga, and G. Müller, J. Appl. Phys. 52, 1998 (1981).

${ }^{2}$ T. Niemeijer, Physica (Utrecht) $\underline{36}, 377$ (1967); S. Katsura, T. Horiguchi, and M. Suzuki, Physica (Utrecht) 46, 67 (1970).

${ }^{3}$ Partial results relevant to the $T=0$ case can be found in B. McCoy, E. Barouch, and D. Abraham, Phys. Rev. A $\underline{4}, 2331$ (1971); H. Vaidya and C. Tracy, Physica (Utrecht) 92, 1 (1978); J. Perk, Phys. Lett. 79A, 1 (1980).

${ }^{4}$ B. McCoy, J. Perk, and R. Shrock, Nucl. Phys. B220 [ FS8], 35, 269 (1983).

${ }^{5}$ J. Lajzerowicz and P. Pfeuty, Phys. Rev. B $\underline{11}, 4560$ (1975).

${ }^{6}$ H. Capel and J. Perk, Physica (Utrecht) 87A, 211 (1977).

${ }^{7}$ E. Barouch and B. McCoy, Phys. Rev. A $\underline{3}, 786$ (1971).

${ }^{8}$ G. Müller, Phys. Rev. B 26, 1311 (1982).

${ }^{9}$ B. McCoy, Phys. Rev. 173, 531 (1968).

${ }^{10}$ A. Luther and I. Peschel, Phys. Rev. B 12, 3908 (1975). 